\title{
Acupuncture and Low Dose Gabapentin Effectively Treat Paclitaxel Induced Peripheral Neuropathy and Prevent Chemotherapy Dose Reduction
}

\author{
Bhatnagar B, Pelser C, Gilmore S, Tait Nand and Bao T* \\ The University of Maryland School of Medicine Marlene and Stewart Greenebaum Cancer Center, Baltimore, MD, USA
}

\begin{abstract}
Peripheral neuropathy is a frequent and well-recognized debilitating complication of taxanes, often necessitating dose-reduction of these effective agents. Here, we present a case in which acupuncture treatments were successfully used in combination with low dose gabapentin to alleviate PIPN symptoms and prevent chemotherapy dose reduction.
\end{abstract}

Keywords: Acupuncture; Peripheral neuropathy; Neoadjuvant chemotherapy

\section{Introduction}

Peripheral neuropathy is a frequent and well-recognized debilitating complication of taxanes, often necessitating dose-reduction of these effective agents $[1,2]$. Within the taxane family, paclitaxel is more commonly associated with the development of peripheral neuropathy than docetaxel $[1,2]$. Management of paclitaxel induced peripheral neuropathy (PIPN) remains a significant clinical challenge. At present, no pharmacologic therapies have been proven to be universally effective [3]. Medications commonly employed to treat PIPN include a diverse class of drugs, such as GABA analogs (i.e. gabapentin and pregabalin), tricyclic anti-depressants, selective serotonin reuptake inhibitors (SSRIs) and opioid analgesics. However, these drugs are often reported as either ineffective or fraught with further complications. As such, non-pharmacologic therapies, such as acupuncture, have generated significant interest in the management of PIPN. Acupuncture has been successfully employed for symptom palliation in patients experiencing peripheral neuropathy secondary to diabetes, HIV and cancer [4-6], and thus presents an attractive treatment option for PIPN. Here, we present a case in which acupuncture treatments were successfully used in combination with low dose gabapentin to alleviate PIPN symptoms and prevent chemotherapy dose reduction.

\section{Case Report}

A 54-year-old postmenopausal African-American female, with no significant past medical history, was diagnosed with stage IIIA (T2N2M0) triple negative breast cancer one month after palpating a left breast mass. She opted to enroll in a clinical trial in which she was randomized to receive neoadjuvant weekly paclitaxel $(80 \mathrm{mg} /$ $\mathrm{m}^{2}$ ) for twelve weeks followed by dose-dense doxorubicin and cyclophosphamide for four cycles.

Following her first two doses of weekly paclitaxel, she developed grade 2 peripheral neuropathy according to the National Cancer Institute-Common Toxicity Criteria (NCI-CTC). Neuropathy symptoms presented in her hands and feet, which she described as a sensation of tingling and numbness that interfered with her daily function. She also reported a painful sensation in her feet, which she rated as 7 out of 10 on a standard pain scale. She was initially prescribed gabapentin $100 \mathrm{mg}$ three times a day for relief of her neuropathic pain symptoms, but was also offered the option of receiving acupuncture if her symptoms persisted. At her next follow-up visit, one week later, by which time she had been taking gabapentin at the prescribed dose for approximately 10 days, she reported a worsening of her neuropathy and elected treatment with acupuncture. For the next two weeks, she was treated with acupuncture per standard protocol once per week as well as taking gabapentin $100 \mathrm{mg}$ three times a day. The standard acupuncture protocol consisted of treatment at the following acupoints: bilateral ear points (shen men, point zero, and two additional auricular acupuncture points where electro-dermal signal was detected); bilateral body acupuncture points LI4, SJ5, LI11, ST40; and Ba Feng located in upper and lower extremities [7]. She experienced an immediate improvement in her neuropathy symptoms right after the acupuncture treatment with pain reduced from 4 to 1 on a ten point scale, and, at each of her subsequent follow-up visits, was found to have an NCI-CTC grade 1 neuropathy with minimal paresthesia and pain. Meanwhile, she continued taking $100 \mathrm{mg}$ of gabapentin three times daily for a total of five weeks, which she discontinued due to dizziness. She continued to receive paclitaxel at full dose and did not require dose reduction or dosing schedule modifications due to neuropathic symptoms. Additionally, she noted that acupuncture also appeared to alleviate her symptom of mild to moderate nausea, also believed to be related to paclitaxel.

After completing twelve weeks of paclitaxel, she proceeded to received four cycles of doxorubicin and cyclophosphamide and had an uncomplicated clinical course without incident. The patient tolerated chemotherapy well, and both gabapentin and acupuncture were discontinued. Her neuropathy symptoms remained minimal during this stage of her treatment. A restaging MRI of the breast following completion of chemotherapy showed significant interval reduction in size of the left breast mass and axillary tail. The patient then underwent left partial mastectomy and left axillary lymph node dissection, which revealed no evidence of invasive ductal carcinoma. To date, she remains in a pathologic complete remission.

\section{Discussion}

This case report suggests that acupuncture in addition to low dose gabapentin may be effective to treat paclitaxel-induced peripheral

*Corresponding author: Ting Bao, Marlene and Stewart Greenebaum Cance Center, University of Maryland School of Medicine, 22 South Greene Street, Baltimore, MD 21201, Tel: 410-328-7225; Fax: 410-328-0805; E-mail: tbao@umm.edu

Received June 15, 2013; Accepted July 01, 2013; Published July 04, 2013

Citation: Bhatnagar B, Pelser C, Gilmore S, Nand T, Bao T (2013) Acupuncture and Low Dose Gabapentin Effectively Treat Paclitaxel Induced Periphera Neuropathy and Prevent Chemotherapy Dose Reduction. Altern Integ Med 2: 126. doi:10.4172/2327-5162.1000126

Copyright: (c) 2013 Bhatnagar B, et al. This is an open-access article distributed under the terms of the Creative Commons Attribution License, which permits unrestricted use, distribution, and reproduction in any medium, provided the original author and source are credited. 
Citation: Bhatnagar B, Pelser C, Gilmore S, Nand T, Bao T (2013) Acupuncture and Low Dose Gabapentin Effectively Treat Paclitaxel Induced Peripheral Neuropathy and Prevent Chemotherapy Dose Reduction. Altern Integ Med 2: 126. doi:10.4172/2327-5162.1000126

neuropathy (PIPN) and to prevent chemotherapy dose reduction. In addition to the direct benefits of symptomatic relief and reduction of neuropathic severity, improvement of PIPN allowed this patient to continue receiving paclitaxel at full-dose, a factor that arguably contributed to her excellent treatment response.

Paclitaxel, and other taxanes, commonly referred to as mictrotubule stabilizing agents (MTSAs) lead to disruption of mitosis in the G2 phase, and exert a broad spectrum of cytotoxic effects in cancers of the breast, ovary and lung [8].

Despite its efficacy, the development of peripheral neuropathy remains the most common cause for paclitaxel dose modifications [1]. PIPN is characterized as an axonal, predominantly, sensory neuropathy $[1,9]$. Symptoms are typically described as numbness and tingling in a "stocking-and-glove" pattern, particularly in the distal lower extremities. Patients may also report intermittent sharp, shooting pains in the legs. Loss of deep tendon reflexes and sensation to pain and temperature are also commonly reported [1]. The incidence of PIPN varies from $2-33 \%$ [10-15] and is based largely on several predisposing risk factors such as dose per cycle, concurrent therapy with other neurotoxic agents, treatment schedule, cumulative dose, duration of infusion and pre-existing neuropathy from other medical conditions such as diabetes $[1,2]$. Furthermore, a reported $58 \%$ of patients develop paclitaxel-associated acute pain syndrome (P-APS). This is a distinct clinical entity, recently characterized by Loprinzi and colleagues, which may also predispose patients to PIPN later in their treatment course [16].

The precise pathogenesis through which paclitaxel exerts its neurotoxic effects is unknown. However, pre-clinical rat models have demonstrated that taxanes disrupt axonal transport, and affect all sensory nerve fibers, particularly large myelinated nerve fibers involved in vibration and proprioception $[8,17,18]$.

Management of PIPN remains challenging considering that commonly used pharmacologic therapies have varying rates of success and often carry their own adverse effects. So far, the only successful clinical trial for treatment of chemotherapy induced peripheral neuropathy (CIPN) treatment clinical trial showed $60 \mathrm{mg}$ daily duloxetine caused a modest 0.7 point pain reduction (on a ten point scale) when compared to placebo, and was also associated with common side effects such as fatigue and nausea [19].

Acupuncture is a well-tolerated; low-risk non-pharmacologic intervention which has been successfully used to treat chemotherapy induced peripheral neuropathy [6,7]. Although the mechanism of acupuncture remains unclear, it has been proposed that acupuncture works through its effect on neurotransmitters and neurohormones. Animal research suggests that acupuncture accomplishes its analgesic effect by stimulating nerves in the muscle, which then relay the signal to the spinal cord, midbrain and hypothalamus-pituitary system. This signaling leads to the release of neurotransmitters and hormones such as endorphins and enkephalins [20-22]. Several other mechanisms have also been suggested to explain the effect of acupuncture analgesia. These include: activation of descending pain inhibiting pathways; deactivation of the limbic system as well as cortical and subcortical regions; cortical cerebral vasodilation causing release of neuropeptide; and inhibition of the release of inflammatory factors [23-27].

In this particular case, the benefit of acupuncture in addition to low dose gabapentin appeared to be two-fold. In addition to providing symptomatic relief, this combination treatment potentially prevented paclitaxel dose reduction, allowing this patient to receive the full benefit of chemotherapy and achieve a pathologic complete response. The role that acupuncture may have in preventing chemotherapy dose reductions is an important consideration in the care of cancer patients receiving neurotoxic chemotherapy regimens. It is also interesting that the patients' PIPN symptoms resolved after 5 weeks of low dose gabapentin in combination with weekly acupuncture treatment. This suggests there may be synergistic effects between the two modalities that warrant further research.

Our case report provides encouraging results regarding the use of acupuncture plus low dose gabapentin in treating PIPN. However, one potential limitation is that it is difficult to discern which component of this patient's treatment played the primary role in palliating her symptoms. The patient did comment that she did not experience any improvement in symptoms during the 10 days of gabapentin treatment before acupuncture was started. Her symptoms greatly improved, however, promptly following initiation of acupuncture treatments. While gabapentin typically takes several weeks to demonstrate its therapeutic effect, we would not expect such a dramatic decline in symptoms on the low dose that this patient received [28]. Importantly, the patient experienced no adverse treatment effects from either gabapentin or acupuncture. Further studies should examine potential synergistic effects of acupuncture combined with low dose antineuropathy agents, with the goal of providing patients with effective treatment with minimal side effects.

\section{Acknowledgements}

TB is a Paul Calabresi scholar (K12 CA126849) and is supported by R2 CA173263.

\section{References}

1. Argyriou AA, Koltzenburg M, Polychronopoulos P, Papapetropoulos S Kalofonos HP (2008) Peripheral nerve damage associated with administration of taxanes in patients with cancer. Crit Rev Oncol Hematol 66: 218-228.

2. Lee JJ, Swain SM (2006) Peripheral neuropathy induced by microtubulestabilizing agents. J Clin Oncol 24: 1633-1642.

3. Pachman DR, Barton DL, Watson JC, Loprinzi CL (2011) Chemotherapy induced peripheral neuropathy: prevention and treatment. Clin Pharmaco Ther 90: 377-387

4. Abuaisha BB, Costanzi JB, Boulton AJ (1998) Acupuncture for the treatment of chronic painful peripheral diabetic neuropathy: a long-term study. Diabetes Res Clin Pract 39: 115-121.

5. Galantino ML, Eke-Okoro ST, Findley TW, Condoluci D (1999) Use of noninvasive electroacupuncture for the treatment of HIV-related peripheral neuropathy: a pilot study. J Altern Complement Med 5: 135-142.

6. Wong R, Sagar S (2006) Acupuncture treatment for chemotherapy-induced peripheral neuropathy--a case series. Acupunct Med 24: 87-91.

7. Bao T, Zhang R, Badros A, Lao L (2011) Acupuncture treatment for bortezomibinduced peripheral neuropathy: a case report. Pain Res Treat 2011: 920807.

8. Rowinsky EK, Donehower RC (1995) Paclitaxel (taxol) N Engl J Med 332 1004-1014.

9. Rowinsky EK, Eisenhauer EA, Chaudhry V, Arbuck SG, Donehower RC (1993) Clinical toxicities encountered with paclitaxel (Taxol). Semin Oncol 20: 1-15.

10. Gradishar WJ, Tjulandin S, Davidson N, Shaw H, Desai N, et al. (2005) Phase III trial of nanoparticle albumin-bound paclitaxel compared with polyethylated castor oil-based paclitaxel in women with breast cancer. J Clin Oncol 23: 7794 7803

11. Jones SE, Erban J, Overmoyer B, Budd GT, Hutchins L, et al. (2005) Randomized phase III study of docetaxel compared with paclitaxel in metastatic breast cancer. J Clin Oncol 23: 5542-5551.

12. Nabholtz JM, Gelmon K, Bontenbal M, Spielmann M, Catimel G, et al. (1996) Multicenter, randomized comparative study of two doses of paclitaxel in patients with metastatic breast cancer. J Clin Oncol 14: 1858-1867. 
Citation: Bhatnagar B, Pelser C, Gilmore S, Nand T, Bao T (2013) Acupuncture and Low Dose Gabapentin Effectively Treat Paclitaxel Induced Peripheral Neuropathy and Prevent Chemotherapy Dose Reduction. Altern Integ Med 2: 126. doi:10.4172/2327-5162.1000126

13. Seidman AD, Tiersten A, Hudis C, Gollub M, Barrett S, et al. (1995) Phase II trial of paclitaxel by 3-hour infusion as initial and salvage chemotherapy for metastatic breast cancer. J Clin Oncol 13: 2575-2581.

14. Smith RE, Brown AM, Mamounas EP, Anderson SJ, Lembersky BC, et al. (1999) Randomized trial of 3-hour versus 24-hour infusion of high-dose paclitaxel in patients with metastatic or locally advanced breast cancer: National Surgical Adjuvant Breast and Bowel Project Protocol B-26. J Clin Oncol 17: 3403-3411.

15. Winer EP, Berry DA, Woolf S, Duggan D, Kornblith A, et al. (2004) Failure of higher-dose paclitaxel to improve outcome in patients with metastatic breast cancer: cancer and leukemia group B trial 9342. J Clin Oncol 22: 2061-2068.

16. Loprinzi CL, Reeves BN, Dakhil SR, Sloan JA, Wolf SL, et al. (2011) Natural history of paclitaxel-associated acute pain syndrome: prospective cohort study NCCTG N08C1. J Clin Oncol 29: 1472-1478.

17. Cavaletti G, Cavalletti E, Montaguti P, Oggioni N, De Negri O, et al. (1997) Effect on the peripheral nervous system of the short-term intravenous administration of paclitaxel in the rat. Neurotoxicology 18: 137-145.

18. Cavaletti G, Cavalletti E, Oggioni N, Sottani C, Minoia C, et al. (2000) Distribution of paclitaxel within the nervous system of the rat after repeated intravenous administration. Neurotoxicology 21: 389-393.

19. Smith EM, Pang H, Cirrincione C, Fleishman S, Paskett ED, et al. (2013) Effect of duloxetine on pain, function, and quality of life among patients with chemotherapy-induced painful peripheral neuropathy: a randomized clinical trial. JAMA 309: 1359-1367.

20. Han JS (2003) Acupuncture: neuropeptide release produced by electrica stimulation of different frequencies. Trends Neurosci 26: 17-22.
21. Berman B (2007) A 60-year-old woman considering acupuncture for knee pain. JAMA 297: 1697-1707.

22. Li A, Lao L, Wang Y, Xin J, Ren K, et al. (2008) Electroacupuncture activates corticotrophin-releasing hormone-containing neurons in the paraventricular nucleus of the hypothalammus to alleviate edema in a rat model of inflammation. BMC Complement Altern Med 8: 20.

23. Hui KK, Liu J, Marina O, Napadow V, Haselgrove C, et al. (2005) The integrated response of the human cerebro-cerebellar and limbic systems to acupuncture stimulation at ST 36 as evidenced by fMRI. Neuroimage 27: 479-496.

24. Jansen G, Lundeberg T, Kjartansson J, Samuelson UE (1989) Acupuncture and sensory neuropeptides increase cutaneous blood flow in rats. Neurosc Lett 97: 305-309.

25. Kashiba H, Ueda $Y$ (1991) Acupuncture to the skin induces release of substance $\mathrm{P}$ and calcitonin gene-related peptide from peripheral terminals of primary sensory neurons in the rat. Am J Chin Med 19: 189-197.

26. Leong RJ, Chernow B (1988) The effects of acupuncture on operative pain and the hormonal responses to stress. Int Anesthesiol Clin 26: 213-217.

27. Li A, Wang Y, Xin J, Lao L, Ren K, et al. (2007) Electroacupuncture suppresses hyperalgesia and spinal Fos expression by activating the descending inhibitory system. Brain Res 1186: 171-179.

28. Rao RD, Michalak JC, Sloan JA, Loprinzi CL, Soori GS, et al. (2007) Efficacy of gabapentin in the management of chemotherapy-induced peripheral neuropathy: a phase 3 randomized, double-blind, placebo-controlled, crossover trial (NOOC3). Cancer 110: 2110-2118. 